\title{
Enumeration of genus-four maps by number of edges
}

\author{
Alexander Mednykh * \\ Sobolev Institute of Mathematics, Novosibirsk State University \\ 630090 Novosibirsk, Russia \\ Alain Giorgetti \\ LIFC, University of Franche-Comté \\ 16 route de Gray, 25030 Besançon CEDEX, France
}

Received 10 December 2009, accepted 4 August 2011, published online 5 October 2011

\begin{abstract}
An explicit form of the ordinary generating function for the number of rooted maps on a closed orientable surface of genus four with a given number of edges is given. An analytical formula for the number of unrooted maps of genus four with a given number of edges is obtained through the number of rooted ones. Both results are new.
\end{abstract}

Keywords: Enumeration, surface, orbifold, rooted map, unrooted map, Fuchsian group.

Math. Subj. Class.: 05C30, 57R18, $20 \mathrm{H1O}$

\section{Introduction}

By a map we mean a 2-cell decomposition of a compact orientable surface. A map is rooted if one of its darts (edge-vertex incidence pairs) is distinguished as a root and unrooted otherwise. Enumeration of maps up to orientation-preserving homeomorphism has attracted a lot of attention in the last decades. Enumeration of rooted maps on the plane by number of edges was done in the pioneering paper by W. T. Tutte [18]. Later, an analogous result for the torus was given by D. Arquès [2]. A structure for the generating function of the number of rooted maps on the surface of given genus $g$ was suggested by E. A. Bender and E. R. Canfield [5]. By making use of their approach they obtained numerical tables of numbers of rooted maps for genera 2 and 3 . The enumeration of rooted maps of given genus by

\footnotetext{
* Supported by the RFBR (grant 06-01-00153), APVV SK-RU-0007-07, FCPK (grant 02.740.11.0457).

E-mail addresses: mednykh@math.nsc.ru (Alexander Mednykh), alain.giorgetti@univ-fcomte.fr (Alain Giorgetti)
} 
number of vertices and faces was done in [3]. Also, a general algorithm to compute the generating function for such numbers was suggested. The results of this algorithm confirm the available data [22] obtained earlier by T. Walsh and A. Lehman for small numbers of edges. Enumeration of unrooted maps is a more complicated problem. For unrooted maps on the oriented sphere a closed analytical counting formula (of the type studied in the present paper) was given by V. A. Liskovets [12]. Counting algorithms suitable for maps either on the oriented sphere or on the general one were independently provided by N. C. Wormald $[23,24]$. A general formula for counting unrooted maps through the number of rooted ones was obtained in [14]. In particular, the problem was completely solved for the torus and the orientable surfaces of genera 2 and 3. Different approaches to counting unrooted maps with prescribed properties were given in the papers [13, 8, 20].

The present paper provides two new results for maps on a closed orientable surface of genus 4 . The first result is an explicit form of the ordinary generating function for the number of rooted maps of genus 4 with a given number of edges. The second result is an explicit analytical formula relating the number of unrooted maps of genus 4 with $n$ edges with numbers of rooted maps of genus $g \leq 4$ and with $e \leq n$ edges.

\section{Orientable combinatorial maps}

By a (combinatorial) map we mean a triple $(D ; R, L)$ composed of a finite set $D$ and two permutations $R$ and $L$, with $L$ satisfying $L^{2}=1$, generating a transitive subgroup of the symmetric group $S_{D}$. The elements of $D$ are called darts and the orbits of $R, L$ and $R L$ are respectively called vertices, edges and faces. Edges of size one are called semiedges. The genus $g$ of a map $M=(D ; R, L)$ is given by $2-2 g=V+E+F-|D|$, where $V$ is the number of vertices, $E$ is the number of edges and $F$ is the number of faces. If $M$ has no semiedges (i.e. if $L$ is fixed-point free), then $|D|=2 E$ and $2-2 g=V-E+F$.

Combinatorial maps describe topological maps on orientable surfaces with a chosen global orientation; the permutation $R$ represents the cyclic order of the edge-ends incident with each vertex encountered by a rotation around that vertex in the direction corresponding to that orientation. Hence they are determined up to orientation-preserving homeomorphisms of the surface leaving the set of vertices, of edges and of faces invariant. This gives rise to the following definition: two maps $\left(D_{1} ; R_{1}, L_{1}\right)$ and $\left(D_{2} ; R_{2}, L_{2}\right)$ are isomorphic, written $\left(D_{1} ; R_{1}, L_{1}\right) \cong\left(D_{2} ; R_{2}, L_{2}\right)$, if there is a bijection $\psi: D_{1} \rightarrow D_{2}$ such that $\psi R_{1}=R_{2} \psi$ and $\psi L_{1}=L_{2} \psi$. The isomorphism class of the map $(D ; R, L)$ is the set of maps $\left(D ; R^{\psi}, L^{\psi}\right)$ such that $\psi \in S_{D}$ is a permutation of $D$. A rooted map is a 4-tuple $(D, r ; R, L)$, where $r \in D$ and $(D ; R, L)$ is a map. The dart $r$ is called the root. Two rooted maps $(D, r ; R, L)$ and $\left(D^{\prime}, r^{\prime} ; R^{\prime}, L^{\prime}\right)$ are isomorphic is there is an isomorphism $(D, R, L)$ onto $\left(D^{\prime}, R^{\prime}, L^{\prime}\right)$ taking root $r$ to root $r^{\prime}$. To each map $M=(D ; R, L)$ there is an associated closed orientable surface (that is, a compact orientable surface without boundary) which can be constructed by associating a 2-cell to each orbit of the permutation $R L$. Hence $M$ can be regarded as a topological map. In turn, any topological map on a closed orientable surface can be realized as a combinatorial map and two topological maps are related by an orientation-preserving homeomorphism if and only if the corresponding combinatorial maps are related by an isomorphism [10].

By enumerating unrooted maps we mean enumerating isomorphism classes of combinatorial maps, which is equivalent to enumerating topological maps up to orientationpreserving homeomorphism. Topological maps correspond to cellular embeddings of 
graphs. Since graphs were generally assumed to be without semiedges, we will interpret "maps on a closed orientable surface of genus $g$ ", or just "genus $g$ orientable maps" as maps without semiedges.

The theory of maps presented in [10] gives a close relationship between maps and subgroups of a certain universal group. Denote by $\Delta=\Delta(\infty, \infty, 2)$ the group $\langle\alpha, \beta| \beta^{2}=$ $1\rangle \cong Z * Z_{2}$. Given a map $(D ; R, L)$, the assignment $\alpha \mapsto R$ and $\beta \mapsto L$ extends to an epimorphism $\Phi: \Delta \rightarrow\langle R, L\rangle$. It follows that $\Delta$ acts on $D$ by $z \cdot x=\Phi(z) x$ for $z \in \Delta$ and $x \in D$. The stabilizer $K \leq \Delta$ of a dart $x \in D$ has index $[\Delta: K]=|D|$. Conversely, each subgroup $K \leq \Delta$ of finite index determines a rooted map $M=(D, r ; R, L)$, where $D$ is the set of left cosets $x K, x \in \Delta, r=K$ is the trivial coset and the action of $R$ and $L$ is defined by left multiplication: $R(x K)=\alpha x K, L(x K)=\beta x K$. Moreover, $M$ has no semiedges if and only if $K$ is torsion-free.

\subsection{Maps on orbifolds}

In this paper we consider maps on orbifolds. This is a new and fruitful idea already used in previous papers $[14,15]$. By an oriented orbifold $O$ we mean an oriented surface $S$ with a discrete subset of points $B=\left\{p_{1}, p_{2}, \ldots\right\}$ such that to each point $p_{i}$ an integer $m_{i} \geq 2$ is assigned. The elements of $B$ will be called branch points and the respective numbers $m_{1}, m_{2}, \ldots, m_{i}, \ldots$ will be called branch indices. If $S$ is a compact connected orientable surface of genus $g$, then $B$ is of finite cardinality $|B|=r$. In this case, the orbifold $O$ is uniquely determined (up to orientation-preserving homeomorphism) by its signature $\left[g ; m_{1}, m_{2}, \ldots, m_{r}\right], 1<m_{1} \leq m_{2} \leq \ldots \leq m_{r}$. Hence we write $O=O\left[g ; m_{1}, m_{2}, \ldots, m_{r}\right]$. The fundamental group $\pi_{1}(O)$ of $O$ is an $F$-group (see [10]) defined by

$$
\begin{aligned}
\pi_{1}(O)= & F\left[g ; m_{1}, m_{2}, \ldots, m_{r}\right] \\
= & \left\langle a_{1}, b_{1}, a_{2}, b_{2}, \ldots, a_{g}, b_{g}, e_{1}, \ldots, e_{r}\right| \\
& \left.\prod_{i=1}^{g}\left[a_{i}, b_{i}\right] \prod_{j=1}^{r} e_{j}=1, e_{1}^{m_{1}}=\cdots=e_{r}^{m_{r}}=1\right\rangle .
\end{aligned}
$$

A map on an orbifold $O$ is a map on the underlying surface $S_{g}$ of genus $g$ satisfying the following three properties:

(P1) if $x \in B$, then $x$ is either an internal point of a face, or a vertex, or an end-point of a semiedge (free end) which is not a vertex,

(P2) each face contains at most one branch point,

(P3) each free end of a semiedge is a branch point and the branch index of this point is 2 .

Maps on orbifolds arise naturally when we take a quotient of an ordinary map on a closed surface by a finite group $G$ of automorphisms. Then the numbers $m_{1}, \ldots, m_{r}$ are the orders of the stabilizers of the faces, vertices and edges under the action of $G$. Note that these stabilizers are always cyclic. Further information on maps on orbifolds can be found in [14] and [15].

An epimorphism $\pi_{1}(O) \rightarrow Z_{\ell}$ onto a cyclic group of order $\ell$ is called order preserving if it preserves the orders of the generators $e_{j}, j=1, \ldots, r$. Equivalently, an order-preserving epimorphism $\pi_{1}(O) \rightarrow Z_{\ell}$ has a torsion-free kernel. We denote by $E p i_{0}\left(\pi_{1}(O), Z_{\ell}\right)$ the number of order-preserving epimorphisms $\pi_{1}(O) \rightarrow Z_{\ell}$. 
For a technical reason it is convenient to modify the signature in the following way. Let

$$
\left[g ; m_{1}, m_{2}, \ldots, m_{r}\right]=[g ; \underbrace{2, \ldots, 2}_{b_{2} \text { times }}, \underbrace{3, \ldots, 3}_{b_{3} \text { times }}, \ldots, \underbrace{\ell, \ldots, \ell}_{b_{\ell} \text { times }}] .
$$

Then we write $\left[g ; 2^{b_{2}}, 3^{b_{3}}, \ldots, \ell^{b_{\ell}}\right]$ rather than $\left[g ; m_{1}, m_{2}, \ldots, m_{r}\right]$ listing only those $j^{b_{j}}$ with $b_{j}>0$.

Denote by $\operatorname{Orb}\left(S_{g} / Z_{\ell}\right)$ the set of $\ell$-tuples $\left[g ; 2^{b_{2}}, 3^{b_{3}}, \ldots, \ell^{b_{\ell}}\right]$ which are the signatures of cyclic orbifolds of type $S_{g} / Z_{\ell}$ for some $S_{g}$ and $Z_{\ell}$. By definition, the fundamental group $\pi_{1}(O)$ is uniquely determined by the signature of the orbifold $O$. Hence, for any $O \in O r b\left(S_{g} / Z_{\ell}\right), O=\left[g ; 2^{b_{2}}, 3^{b_{3}}, \ldots, \ell^{b_{\ell}}\right]$, the group $\pi_{1}(O)$ is well defined.

\section{Rooted map enumeration}

Let $Q_{g}(z)=\sum_{n \geq 0} N_{g}(n) z^{n}$ be the ordinary generating function counting the number $N_{g}(n)$ of rooted maps on the orientable surface of genus $g$ by number of edges (the exponent of $z$ ). This generating function satisfies an equation system presented by $\mathrm{E}$. A. Bender and E. R. Canfield in [4]. Given a genus $g$, a closed expression for $Q_{g}(z)$ can in principle be computed from this equation system by induction. However the computational complexity is so high that up to 1998 exact solutions where only known for the first four genera, from 0 to 3 . A common pattern for all the $Q_{g}(z)$, where $g$ ranges over the positive integers, was proposed in [5]. Each $Q_{g}(z)$ is a rational function of a quadratic parameter of $z$, but this pattern leaves a polynomial of this parameter unknown. An upper bound for the polynomial degree is conjectured but not proved. The first proof of a more precise pattern, with a maximal degree for each unknown polynomial, is due to D. Arquès and the second author [3, 9], for the more general case of counting by number of vertices and faces. This section presents new results derived from this former work by focusing on counting by number of edges.

\subsection{Generating functions counting rooted maps}

The following result proves the conjecture in [5] and is an easy consequence of Theorem 1 of [3].

Theorem 3.1. For any positive integer $g$, the ordinary generating function $Q_{g}(z)$ counting rooted maps on a closed orientable surface of genus $g$ by number of edges (exponent of $z$ ) can be written

$$
Q_{g}(z)=z^{2 g}(1-3 m)^{-2}(1-2 m)^{4-5 g}(1-6 m)^{3-5 g} P_{g}(m),
$$

where $m=\frac{1-\sqrt{1-12 z}}{6}$ and $P_{g}(m)$ is a polynomial of $m$ of degree less than or equal to $6 g-6$.

The explicit formulae for polynomials $P_{g}(m), g=0,1,2,3$ can be derived from the papers [19], [2], [5] and [9], respectively. The first step to counting unrooted genus-four maps by number of edges is given by the following proposition.

Proposition 3.2. The polynomial $P_{g}$ in Theorem 3.1 for $g=4$ is given by the formula 


$$
\begin{aligned}
P_{4}(m)= & 9(1-2 m)^{6} \\
& \left(41956066368 m^{12}-107657028288 m^{11}+128766120048 m^{10}\right. \\
& -95026128096 m^{9}+48202134300 m^{8}-17709582732 m^{7} \\
& +4855070265 m^{6}-1025233956 m^{5}+178608786 m^{4} \\
& \left.-28633200 m^{3}+4245462 m^{2}-465894 m+25025\right) .
\end{aligned}
$$

Proof. Theorem 5.1, Relation (6.1) and Propositions 6.1 and 6.2 from [9] make it possible to compute $P_{g}$ from polynomials of lower degree. This result has been computed by a software developed by the second author in his $\mathrm{Ph} . \mathrm{D}$. thesis. The software does not directly compute the polynomials but rather the generating functions enumerating rooted maps by genus, along the principles presented in [5] and detailed in [9]. It has not been designed for efficiency but it successfully computes the generating functions for rooted maps up to genus 4. The genus-four formula was computed in less than one minute on an Intel Pentium at 1.4 $\mathrm{GHz}$ with $1.25 \mathrm{~Gb}$ of memory. In accordance with Theorem 3.1, the polynomial computed for $P_{4}$ is indeed of degree $6 g-6=18$.

\subsection{Counting rooted genus-four maps by number of edges}

Theorem 3.3. The ordinary generating function $Q_{4}(z)$ counting rooted maps on a closed orientable surface of genus four by number of edges (the exponent of $z$ ) is

$$
\begin{aligned}
Q_{4}(z)= & 9 z^{8}(1-3 m)^{-2}(1-2 m)^{-10}(1-6 m)^{-17} \\
& \left(41956066368 m^{12}-107657028288 m^{11}+128766120048 m^{10}\right. \\
& -95026128096 m^{9}+48202134300 m^{8}-17709582732 m^{7} \\
& +4855070265 m^{6}-1025233956 m^{5}+178608786 m^{4} \\
& \left.-28633200 m^{3}+4245462 m^{2}-465894 m+25025\right)
\end{aligned}
$$

where $m=\frac{1-\sqrt{1-12 z}}{6}$.

Proof. Formula (3.2) follows from Theorem 3.1 and Proposition 3.2.

Table 1 presents the coefficients of $Q_{g}(z)=\sum_{n>0} N_{g}(n) z^{n}$. The first seven coefficients correspond to the ones found by Timothy Walsh in his Ph.D. thesis [21]. The remaining coefficients are new.

\section{Unrooted map enumeration}

\subsection{Counting unrooted maps through rooted maps on orbifolds}

The following theorem is the main result of [14].

Theorem 4.1. The number $U_{g}(e)$ of unrooted maps with e edges on a closed orientable surface of genus $g$ is given by the formula

$$
U_{g}(e)=\frac{1}{2 e} \sum_{\ell m=2 e} \sum_{O \in O r b\left(S_{g} / Z_{\ell}\right)} \operatorname{Epi}_{0}\left(\pi_{1}(O), Z_{\ell}\right) \nu_{O}(m),
$$

where $\nu_{O}(m)$ is the number of rooted maps with $m$ darts on the orbifold $O$. 


\begin{tabular}{r|l}
$n$ & The number $N_{4}(n)$ of rooted maps of genus 4 with $n$ edges \\
\hline 8 & 225225 \\
9 & 24635754 \\
10 & 1495900107 \\
11 & 66519597474 \\
12 & 2416610807964 \\
13 & 75981252764664 \\
14 & 2141204115631518 \\
15 & 5535267009315660 \\
16 & 1334226671709010578 \\
17 & 30347730709395639732 \\
18 & 657304672067357799042 \\
19 & 13652607304062788395788 \\
20 & 273469313030628783700080 \\
21 & 5306599156694095573465824 \\
22 & 100128328831437989131706976 \\
23 & 1842794650155970906232185656 \\
24 & 33167202398202989127880734894 \\
25 & 585079650671639944950451625580 \\
26 & 10134917623511547808118654370114 \\
27 & 172678013694177771071548169002188 \\
28 & 2897912714075648947715005321906392 \\
29 & 47963145773909943419634526762950192 \\
30 & 783757995914247522485178250636927380 \\
31 & 12657015244648210693716700196736399336 \\
32 & 202177082281879102698899470748726765316 \\
33 & 3196834110175421253323791465873251739560 \\
34 & 50072299181065185108291501010224952255668 \\
35 & 777384663760023780739632793721755383049272 \\
36 & 11969638731261482998116895312223651253180480 \\
37 & 182875502596501323216343759769794526714561664 \\
38 & 2773716775724835345230901154059649970954877396 \\
39 & 41781661724286164921640221635213792280118832368 \\
40 & 625310196714095279935937237998816771771464314790 \\
41 & 9301365625304817339752604766781541863133507845340 \\
42 & 137556789724353166312824029682866215741796911453698 \\
43 & 2023172807939725017933640132814869413798020476575564 \\
44 & 29601998835280343256197223863418277211551813053748872 \\
45 & 430981509422356688373368386557125320381885703792230800
\end{tabular}

Table 1: Enumeration of rooted maps of genus 4 by number of edges.

The number of rooted maps on the orbifold $O=O\left[g ; 2^{b_{2}}, \ldots, \ell^{b_{\ell}}\right]$ can be expressed through the number $N_{g}(n)$ of rooted maps on genus $g$ surface by the following proposition given in [14].

Proposition 4.2. Let $O=O\left[g ; 2^{b_{2}}, \ldots, \ell^{b_{\ell}}\right]$ be an orbifold, $b_{i} \geq 0$ for $i=2, \ldots, \ell$. Let 
$N_{g}(n)$ be the number of rooted maps of genus $g$ with $n$ edges. Then the number of rooted maps $\nu_{O}(m)$ with $m$ darts on the orbifold $O$ is

$$
\nu_{O}(m)=\sum_{s=0}^{b_{2}}\left(\begin{array}{c}
m \\
s
\end{array}\right)\left(\begin{array}{c}
\frac{m-s}{2}+2-2 g \\
b_{2}-s, b_{3}, \ldots, b_{\ell}
\end{array}\right) N_{g}((m-s) / 2)
$$

where $N_{g}(n)=0$ if $n$ is not an integer.

Denote by $\mu(n), \phi(n)$ and $\Phi(x, n)$ the Möbius, Euler and von Sterneck functions [1, 16]. The relationship between them is given by the formula

$$
\Phi(x, n)=\frac{\phi(n)}{\phi\left(\frac{n}{(x, n)}\right)} \mu\left(\frac{n}{(x, n)}\right)
$$

where $(x, n)$ is the greatest common divisor of $x$ and $n$. It was shown by O. Hölder that $\Phi(x, n)$ coincides with the Ramanujan sum $\sum_{\substack{1 \leq k \leq n \\(k, n)=1}} \exp \left(\frac{2 i k x}{n}\right)$. For the proof, see [1, p.164] and [16].

Recall that the Jordan multiplicative function $\phi_{k}(n)$ of order $k$ can be defined (for more information see [7, p.199], [11, 17]) as

$$
\phi_{k}(n)=\sum_{d \mid n} \mu\left(\frac{n}{d}\right) d^{k}
$$

From [14] we have the following proposition.

Proposition 4.3. Let $O=O\left[g ; m_{1}, \ldots, m_{r}\right]$ be an orbifold of signature $\left(g ; m_{1}, \ldots, m_{r}\right)$. Denote by $m=l \mathrm{~cm}\left(m_{1}, \ldots, m_{r}\right)$ the least common multiple of $m_{1}, \ldots, m_{r}$ and let $\ell$ be a multiple of $m$. Then the number of order-preserving epimorphisms of the orbifold fundamental group $\pi_{1}(O)$ onto a cyclic group $Z_{\ell}$ is given by the formula

$$
E p i_{0}\left(\pi_{1}(O), Z_{\ell}\right)=m^{2 g} \phi_{2 g}(\ell / m) E\left(m_{1}, m_{2}, \ldots, m_{r}\right),
$$

where

$$
E\left(m_{1}, m_{2}, \ldots, m_{r}\right)=\frac{1}{m} \sum_{k=1}^{m} \Phi\left(k, m_{1}\right) \cdot \Phi\left(k, m_{2}\right) \cdot \ldots \cdot \Phi\left(k, m_{r}\right)
$$

$\phi_{2 g}(\ell)$ is the Jordan multiplicative function of order $2 g$, and $\Phi(k, m)$ is the von Sterneck function. 


\subsection{Counting unrooted genus-four maps by number of edges}

Theorem 4.4. The number $U_{4}(e)$ of unrooted maps on a closed orientable surface of genus four counted by the number of edges $e$ is given by the formula

$$
\begin{gathered}
\quad \frac{1}{2 e}\left(N_{4}(e)+16 \nu_{\left[2 ; 2^{2}\right]}(e)+4 \nu_{\left[1 ; 2^{6}\right]}(e)+\nu_{\left[0,2^{10}\right]}(e)\right. \\
+80 N_{2}(e / 3)+18 \nu_{\left[1 ; 3^{3}\right]}(2 e / 3)+22 \nu_{\left[0 ; 3^{6}\right]}(2 e / 3) \\
+32 \nu_{\left[1 ; 4^{2}\right]}(e / 2)+8 \nu_{\left[0 ; 2,4^{4}\right]}(e / 2)+2 \nu_{\left[0 ; 2^{4}, 4^{2}\right]}(e / 2) \\
+52 \nu_{\left[0 ; 5^{4}\right]}(2 e / 5)+32 \nu_{\left[1 ; 2^{2}\right]}(e / 3)+2 \nu_{\left[0 ; 2,6^{3}\right]}(e / 3) \\
+2 \nu_{\left[0 ; 2^{2}, 3^{3}\right]}(e / 3)+6 \nu_{\left[0 ; 3^{2}, 6^{2}\right]}(e / 3)+2 \nu_{\left[0 ; 2^{3}, 3,6\right]}(e / 3) \\
+4 \nu_{\left[0 ; 2^{2}, 8^{2}\right]}(e / 4)+18 \nu_{\left[0 ; 9^{3}\right]}(2 e / 9)+12 \nu_{\left[0 ; 5,10^{2}\right]}(e / 5) \\
+4 \nu_{\left[0 ; 2^{2}, 5^{2}\right]}(e / 5)+4 \nu_{\left[0 ; 3,12^{2}\right]}(e / 6)+4 \nu_{[0 ; 4,6,12]}(e / 6) \\
\left.+8 \nu_{[0 ; 3,5,15]}(2 e / 15)+8 \nu_{\left[0 ; 2,16^{2}\right]}(e / 8)+6 \nu_{[0 ; 2,9,18]}(e / 9)\right)
\end{gathered}
$$

where $\nu_{O}(m)$ is defined in Proposition 4.2 and $N_{g}(e)$ is the number of rooted maps of genus $g$ with e edges.

Proof. O.V. Bogopol'skii [6] described all possible signatures of orbifolds of the type $S_{4} / G$, where $S_{4}$ is a surface of genus four and $G$ is a finite group of homeomorphisms acting on $S_{4}$. In particular, $G=Z_{\ell}$ is cyclic of order $\ell$ only for $\ell=1,2,3,4,5,6,8,9,10$, $12,15,16$ and 18 .

From this observation and Proposition 4.3 we get the following lemma.

Lemma 4.5. Let $O \in \operatorname{Orb}\left(S_{4} / Z_{\ell}\right)$. Then one of the following cases occurs:

(1) $\ell=1: O=O[4 ; \emptyset]$ with $\operatorname{Epi}_{0}\left(\pi_{1}(O), Z_{\ell}\right)=1$;

(2) $\ell=2: O=O\left[2 ; 2^{2}\right], O\left[1 ; 2^{6}\right], O\left[0 ; 2^{10}\right]$ with $\operatorname{Epi}_{0}\left(\pi_{1}(O), Z_{\ell}\right)=16,4,1$;

(3) $\ell=3: O=O[2 ; \emptyset], O\left[1 ; 3^{3}\right], O\left[0 ; 3^{6}\right]$ with $\operatorname{Epi}_{0}\left(\pi_{1}(O), Z_{\ell}\right)=80,18,22$;

(4) $\ell=4: O=O\left[1 ; 4^{2}\right], O\left[0 ; 2,4^{4}\right], O\left[0 ; 2^{4}, 4^{2}\right]$ with $E p i_{0}\left(\pi_{1}(O), Z_{\ell}\right)=32,8,2$;

(5) $\ell=5: O=O\left[0 ; 5^{4}\right]$ with $\operatorname{Epi}_{0}\left(\pi_{1}(O), Z_{\ell}\right)=52$;

(6) $\ell=6: O=O\left[1 ; 2^{2}\right], O\left[0 ; 2,6^{3}\right], O\left[0 ; 2^{2}, 3^{3}\right], O\left[0 ; 3^{2}, 6^{2}\right], O\left[0 ; 2^{3}, 3,6\right]$ with $\operatorname{Epi}_{0}\left(\pi_{1}(O), Z_{\ell}\right)=32,2,2,6,2$;

(7) $\ell=8,9: O=O\left[0 ; 2^{2}, 8^{2}\right], O\left[0 ; 9^{3}\right]$ with Epi $i_{0}\left(\pi_{1}(O), Z_{\ell}\right)=4,18$;

(8) $\ell=10: O=O\left[0 ; 5,10^{2}\right], O\left[0 ; 2^{2}, 5^{2}\right]$ with Epi $i_{0}\left(\pi_{1}(O), Z_{\ell}\right)=12$, 4 ;

(9) $\ell=12: O=O\left[0 ; 3,12^{2}\right], O[0 ; 4,6,12]$ with $\operatorname{Epi}_{0}\left(\pi_{1}(O), Z_{\ell}\right)=4,4$;

(10) $\ell=15,16,18: O=O[0 ; 3,5,15], O\left[0 ; 2,16^{2}\right], O[0 ; 2,9,18]$ with $\operatorname{Epi}_{0}\left(\pi_{1}(O), Z_{\ell}\right)=8,8,6$.

Now, the theorem follows from Lemma 4.5 and Theorem 4.1.

We present the numbers thus obtained in Table 2. 


\begin{tabular}{r|l}
$n$ & The number $U_{4}(n)$ of unrooted maps of genus 4 with $n$ edges \\
\hline 8 & 14118 \\
9 & 1369446 \\
10 & 74803564 \\
11 & 3023693380 \\
12 & 100692692173 \\
13 & 2922359760376 \\
14 & 76471600288836 \\
15 & 1845089145736960 \\
16 & 41694584320696782 \\
17 & 892580319444417876 \\
18 & 18258463136626650660 \\
19 & 359279139700128276168 \\
20 & 6836732826365623258492 \\
21 & 126347598971804884131800 \\
22 & 2275643837092089686415858 \\
23 & 40060753264325317709454720 \\
24 & 690983383296198882647616692 \\
25 & 11701593013434174490416914028 \\
26 & 194902261990612930685627941344 \\
27 & 3197740994336653065511697474864 \\
28 & 51748441322779568341478022803550 \\
29 & 826950789205344386488852660387184 \\
30 & 13062633265237461036677963280146184 \\
31 & 204145407171745343738289312062076704 \\
32 & 3159016910654361022421358641441865404 \\
33 & 48436880457203352503593806713722630064 \\
34 & 736357340898017428826654622692598290184 \\
35 & 11105495196571768299465860194739273233104 \\
36 & 166244982378631708320492461115910055656280 \\
37 & 2471290575628396259735279442756616067154240 \\
38 & 36496273364800465069054923670966215780880134 \\
39 & 535662329798540575919393860220780811735355616 \\
40 & 7816377458926190999203023224069385048268971894 \\
41 & 113431288113473382192120378978151871847586693068 \\
42 & 1637580830051823408486063085325625200796356325376 \\
43 & 23525265208601453696903044755726884736237505268568 \\
44 & 336386350400912991547696743715303742977503309390766 \\
45 & 4788683438026185426370763920603749547029983530699104 \\
&
\end{tabular}

Table 2: Enumeration of unrooted maps of genus 4 by number of edges.

\section{Acknowledgments}

The idea of writing this paper was born as a result of a discussion between Valery Liskovets, Roman Nedela, Timothy Walsh and the first author during the conference GEMS' 09 held in Tále, Slovakia, 28 June - 03 July, 2009. We thank Prof. T. Walsh for his stimulating 
discussions by e-mail and for his corrections of an early version of the present text. The authors are also grateful to the anonymous referees for helpful comments and suggestions.

\section{References}

[1] T. M. Apostol, Introduction to analytical number theory, Springer-Verlag, Berlin-New York, 1976.

[2] D. Arquès, Relations fonctionnelles et dénombrement des cartes pointées sur le tore, J. Comb. Theory, Ser. B 43 (1987), 253-274.

[3] D. Arquès and A. Giorgetti, Énumération des cartes pointées de genre quelconque en fonction des nombres de sommets et de faces, J. Comb. Theory, Ser. B 77 (1999), 1-24.

[4] E. A. Bender and E. R. Canfield, The asymptotic number of rooted maps on a surface, J. Comb. Theory, Ser. A 43 (1986), 244-257.

[5] E. A. Bender and E. R. Canfield. The number of rooted maps on an orientable surface, J. Comb. Theory, Ser. B $\mathbf{5 3}$ (1991), 293-299.

[6] O. V. Bogopol'skii, Classifying the action of finite groups on oriented surface of genus 4, Siberian Adv. Math., 7 (1997), 9-38.

[7] L. Comtet, Advanced Combinatorics, Reidel, 1974.

[8] E. Fusy, Counting Unrooted Maps Using Tree Decomposition, Séminaire Lotharingien de Combinatoire 54A (2007), Article B54Al, 44 pp.

[9] A. Giorgetti, Combinatoire bijective et énumérative des cartes pointées sur une surface, $\mathrm{PhD}$ thesis, Université de Marne-la-Vallée, Institut Gaspard Monge, 1998.

[10] G. A. Jones and D. Singerman, Theory of maps on orientable surfaces, Proc. London Math. Soc. 37 (1978), 273-307.

[11] C. Jordan, Traité des substitutions et des équations algébriques, Réimpression de l'orig. 1870, Edition J. Gabay, Paris, 1989.

[12] V. A. Liskovets, A census of nonisomorphic planar maps, in: L. Lovász and V. T. Sós (eds), Algebraic methods in graph theory, volume 25 of Colloq. Math. Soc. János Bolyai, NorthHolland, Amsterdam-New York, 1981, 479-494.

[13] V. A. Liskovets and T. R. S. Walsh, The enumeration of non-isomorphic 2-connected planar maps. Canad J. Math. 35 (1983), 417-435.

[14] A. Mednykh and R. Nedela, Enumeration of unrooted maps of a given genus, J. Comb. Theory, Ser. B 96 (2006), 706-729.

[15] A. Mednykh and R. Nedela, Enumeration of unrooted hypermaps, Electronic Notes in Discrete Mathematics 28 (2007), 207-214.

[16] C. A. Nicol and H. S. Vandiver, A von Sterneck arithmetical function and restricted partitions with respect to modulus, Proc. Nat. Acad. Sci. USA 40 (1954), 825-835.

[17] J. Schulte, Über die Jordansche Verallgemeinerung der Eulerschen Funktion, Result. Math. 36 (1999), 354-364.

[18] W. T. Tutte, A census of planar maps, Canad. J. Math. 15 (1963), 249-271.

[19] W. T. Tutte, On the enumeration of planar maps, Bull. Amer. Math. Soc. 74 (1968), 64-74.

[20] S. Vidal and M. Petitot, Counting Rooted and Unrooted Triangular Maps, J. Nonlinear Syst. Appl. 1 (2010), 51-57.

[21] T. R. S. Walsh, Combinatorial enumeration of non-planar maps, $\mathrm{PhD}$ thesis, University of Toronto, 1971. 
[22] T. R. S. Walsh and A. B. Lehman, Counting rooted maps by genus I, J. Comb. Theory, Ser. B 13 (1972), 192-218.

[23] N. C. Wormald, Counting unrooted planar maps, Discrete Math. 36 (1981), 205-225.

[24] N. C. Wormald, On the number of planar maps, Canad. J. Math. 33 (1981), 1-11. 